\title{
Multiple-Weight Recurrent Neural Networks
}

\author{
Zhu Cao $^{1 *}$, Linlin Wang ${ }^{1 *}$ and Gerard de Melo ${ }^{2}$ \\ ${ }^{1}$ IIIS, Tsinghua University, Beijing, China \\ ${ }^{2}$ Rutgers University, New Brunswick, NJ, USA \\ \{cao-z13, 11-wang13\}@mails.tsinghua.edu.cn, gdm@demelo.org
}

\begin{abstract}
Recurrent neural networks (RNNs) have enjoyed great success in speech recognition, natural language processing, etc. Many variants of RNNs have been proposed, including vanilla RNNs, LSTMs, and GRUs. However, current architectures are not particularly adept at dealing with tasks involving multi-faceted contents, i.e., data with a bimodal or multimodal distribution. In this work, we solve this problem by proposing Multiple-Weight RNNs and LSTMs, which rely on multiple weight matrices to better mimic the human ability of switching between contexts. We present a framework for adapting RNN-based models and analyze the properties of this approach. Our detailed experimental results show that our model outperforms previous work across a range of different tasks and datasets.
\end{abstract}

\section{Introduction}

Deep learning has led to remarkable advances across a diverse range of machine learning tasks in recent years [Bengio and others, 2009]. Various kinds of deep network architectures have been proposed, including Multi-Layer Neural Networks, Convolutional Neural Networks (CNN), and Recurrent Neural Networks (RNNs). RNN-style networks have emerged as the dominant approach for sequential data such as natural language, achieving great success on tasks such as speech recognition, language modeling, and text generation.

Over the years, a number of variants of RNNs have been proposed. The well-known Long Short Term Memory (LSTM) networks [Hochreiter and Schmidhuber, 1997] were designed with the goal of mitigating the vanishing and exploding gradient problems [Bengio et al., 1994; Hochreiter and Schmidhuber, 1997]. LSTM cells are able to capture longer-range associations by aggregating increasing amounts of information. They have enjoyed remarkable success on highly non-trivial tasks such as machine translation and relation extraction.

LSTMs rely on information control mechanisms in the form of gates that modulate how the state is updated, given

\footnotetext{
${ }^{*}$ These authors contributed equally to this work.
}

values computed from the current input and the previous hidden state. Different gating structures and information flow mechanisms can be considered. Cho et al. [2014] presented Gated Recurrent Units (GRUs), which are able to adaptively collect dependencies at different time scales. GRUs, too, have been successfully applied across a range of tasks, including machine translation. A number of minor variations of LSTMs [Greff et al., 2016] and GRUs [Jozefowicz et al., 2015] have been studied. The Minimal Gated Unit (MGU) architecture [Zhou et al., 2016] relies on a simplified model with just a single gate. In contrast, the recent Multi-Function Recurrent Units (Mu-FuRUs) adopt an elaborate gating mechanism that allows for additional differentiable functions as composition operations [Weissenborn and Rocktäschel, 2016]. Overall, we see that it remains important to explore the space of design choices for information flow and gating mechanisms in recurrent neural networks.

In this paper, we study such gating mechanisms focusing in particular on the important challenge of how to develop models that perform robustly on challenging multi-faceted tasks. Humans are naturally adept at adapting to different kinds of tasks. For instance, we can rapidly switch between different levels of formality in conversation, interacting with our colleague using colloquial slang in one moment, while speaking formally to a superior in the next. To this end, we develop a more adaptive RNN-style architecture that consists of multiple weight matrices so as to enhance the model's ability of handling heterogeneous types of content across different test instances.

The main contributions of this work are as follows:

1. We propose the novel paradigm of multi-weight matrix RNNs, seeking to mimic the human ability of separating and switching between different subtasks or problem facets and addressing each of them appropriately.

2. This paradigm is formalized as a framework that is readily applicable to numerous RNN-family network architectures. We analyze this framework both at an abstract level and empirically.

3. Our extensive experimental evaluation on three tasks, namely language modeling, cloze tests, and characterlevel word embeddings, shows that our model is successfully able to exploit the multi-faceted nature of the data, surpassing previous models on these tasks and obtaining new state-of-the-art results on some. 


\section{Related Work}

Recurrent neural networks, a form of neural networks with self-recurrent connections, were extensively studied during the 1990s [Fausett, 1994]. It was soon observed that ordinary recurrent neural networks, in training, suffer from the problems of exploding and vanishing gradients [Bengio et al., 1994]. Hence, more elaborate RNN networks were designed so as to overcome these drawbacks. LSTM networks sought to achieve this by relying on three gates that control the information flow [Hochreiter and Schmidhuber, 1997]. The more recent Gated Recurrent Unit (GRU) networks rely on two gates to capture the dependencies of different time scales adaptively [Cho et al., 2014]. Apart from these wellestablished units, a number of extensions and variants have been put forth, including Structurally Constrained Recurrent Networks (SCRNs) [Mikolov et al., 2014], IRNNs [Le et al., 2015], and Minimal Gated Units (MGU) [Zhou et al., 2016]. Multi-Function Recurrent Units (Mu-FuRUs) adapt the gating mechanism by allowing it to select among a set of suitable composition functions [Weissenborn and Rocktäschel, 2016]. Our work presents a framework showing how many of these RNN-style algorithms can additionally operate using multiple weight matrices.

As for the tasks we consider, important works for language modeling include the recurrent neural network language model (RNNLM) [Mikolov et al., 2010], which addressed the problem that previous feedforward networks depended on fixed length contexts, which need to be specified ad hoc before training. LSTM-based language models have also achieved strong results on language modeling due to their superior capability of capturing longer-term dependencies [Hochreiter and Schmidhuber, 1997]. For dialogue systems, contextual information and dialogue interactions between speakers are important signals. Thus, several contextual language models have been proposed, such as a contextdependent RNN language model [Mikolov and Zweig, 2012], larger-context language models [Wang and Cho, 2015], and two state-of-the-art dialogue context language models [Liu and Lane, 2017]. A significant challenge for word embeddings is how to address the problem of rare and out-ofvocabulary words. Here, character-level information provides significant linguistic features for learning a good representation not only for rare words but also for morphologically complex words.

Finally, we contrast our work with other lines of works sharing seemingly similar goals. In multi-task learning [Caruana, 1997], it is known from which task each input comes, whereas in our setting, there is a single task with multi-faceted data, so the system autonomously needs to learn to choose different weight matrices. Another related task is domain adaptation [Glorot et al., 2011]. Here, training occurs on one domain and then the trained model is applied (only) to another domain. Our model, in contrast, is simultaneously applied to heterogeneous multi-faceted data, without knowledge of which domains a given input instance originates from. Our architecture needs to learn on its own how to identify and handle relevant domains or facets, which can be mixed even within a single instance. Our idea of relying

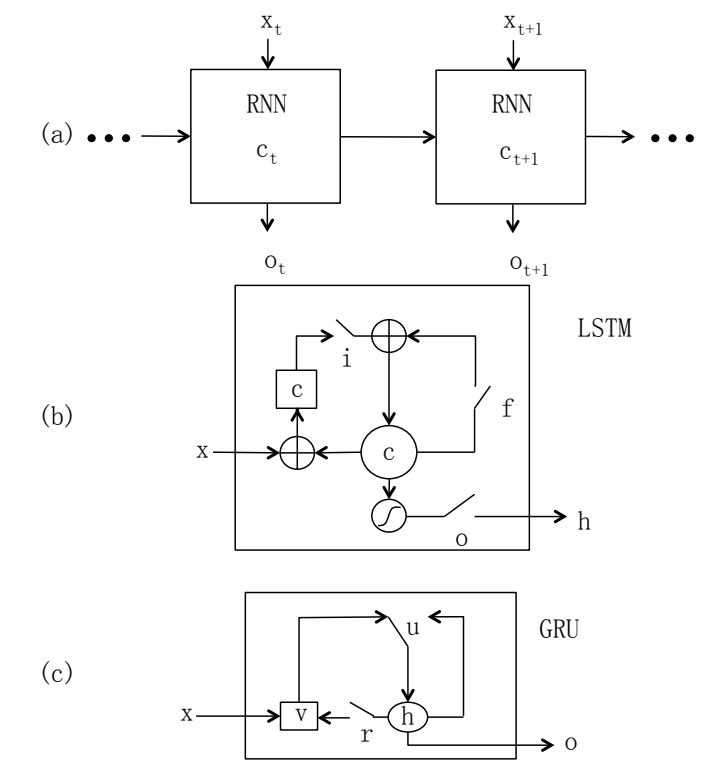

Figure 1: The structures of naïve RNN, LSTM, and GRU.

on multiple weight matrices is also distinct from the notion of ensemble methods, which also duplicate parts the model, and are often used for increased accuracy and robustness of neural networks. We describe the differences in Section 3.2. Finally, the differences to attention models are analyzed in Section 4.4.

\section{Multi-Weight RNN Model}

In this section, we describe the details of our proposed model. We will first review model structures from past work, and then introduce the key innovations of our framework, which allows us to modify these previous architectures.

\subsection{Basic RNN-based Models}

An RNN-type structure consists of RNN cells of the form illustrated in Fig. 1(a). Such cells can be described in terms of their update equations as follows:

$$
\begin{aligned}
& c_{t}=f\left(W_{\mathrm{h}} x_{t}+U_{\mathrm{h}} c_{t-1}+b\right), \\
& o_{t}=g\left(W_{\mathrm{y}} c_{t}+b_{\mathrm{y}}\right) .
\end{aligned}
$$

Here the subscript $t$ stands for the $t$-th timestamp, $x_{t}, c_{t}, o_{t}$ are the input vector, the state vector, and the output vector, respectively, and $f, g$ are activation functions. For a given sequence of inputs, the repetitive application of this procedure can be described in terms of connected chains of RNNs, as illustrated in Fig. 1(a).

A naïve RNN cell computes a new state vector at every step using matrix multiplications and a non-linearity such as tanh. To overcome the naïve RNN's inability to store long-range information, several enhanced RNN cells have been proposed, among which the most well-known ones are Long Short Term Memory (LSTM) [Hochreiter and Schmidhuber, 1997] and Gated Recurrent Unit (GRU) [Cho et al., 2014] cells. An LSTM cell is illustrated in Fig. 1(b) and can be described by 
the following equations:

$$
\begin{aligned}
i_{t} & =\sigma\left(W_{\mathrm{ix}} x_{t}+W_{\mathrm{ih}} h_{t-1}+b_{\mathrm{i}}\right), \\
f_{t} & =\sigma\left(W_{\mathrm{fx}} x_{t}+W_{\mathrm{fh}} h_{t-1}+b_{\mathrm{f}}\right), \\
o_{t} & =\sigma\left(W_{\mathrm{ox}} x_{t}+W_{\mathrm{oh}} h_{t-1}+b_{\mathrm{o}}\right), \\
c_{t} & =f_{t} \odot c_{t-1}+i_{t} \odot \tanh \left(W_{\mathrm{cx}} x_{t}+W_{\mathrm{ch}} h_{t-1}+b\right), \\
h_{t} & =o_{t} \odot c_{t} .
\end{aligned}
$$

Here, as in a basic RNN cell, $t$ stands for the $t$-th timestamp, $x_{t}, c_{t}, h_{t}$ are the input vector, state vector, and hidden vector, respectively, and $i_{t}, f_{t}, o_{t}$ refer to the input gate, forget gate, and output gate, respectively. Finally, the $W$ are weight matrices, $b$ are bias terms, and $\sigma$ is the sigmoid function.

Compared to LSTMs, GRUs do not need any intermediate vector $c_{t}$. The structure of a GRU is illustrated in Fig. 1(c) and can be expressed as follows:

$$
\begin{aligned}
r_{t} & =\sigma\left(W_{\mathrm{rx}} x_{t}+W_{\mathrm{rh}} h_{t-1}+b_{\mathrm{r}}\right), \\
u_{t} & =\sigma\left(W_{\mathrm{ux}} x_{t}+W_{\mathrm{uh}} h_{t-1}+b_{\mathrm{u}}\right), \\
v_{t} & =\tanh \left[W_{\mathrm{vx}} x_{t}+W_{\mathrm{vr}}\left(r_{t} \odot h_{t-1}\right)+b_{\mathrm{v}}\right], \\
o_{t} & =h_{t}=u_{t} \odot h_{t-1}+\left(1-u_{t}\right) \odot v_{t} .
\end{aligned}
$$

Here, $x_{t}, h_{t}, v_{t}, o_{t}$ are the input vector, the original activation vector, the new activation vector, and the output vector, respectively, while $u_{t}, r_{t}$ refer to the update gate and reset gate, respectively. It has been found that GRUs and LSTMs achieve somewhat similar accuracies, but that GRUs are faster in terms of the convergence time and required training epochs [Chung et al., 2014].

\subsection{Our Model}

Having introduced previous RNN-type models, we now present our novel multi-weight architecture. The central idea in our proposal is to allow for multiple matrices that may update the state vector. During training, the model will then learn to adaptively decide on which of these to rely on, based on the current input and the previous state at every time step. Technically, we replace each occurrence of $\tanh (W x+b)$ by a weighted sum

$$
\sum_{i} p_{i} \tanh \left(W_{i} x+b_{i}\right)
$$

where $\tanh (\cdot)$ is the hypertangent function. Note that we are not combining the matrices $W_{i}$ as $\tanh \left(\sum W_{i}\right)$, since that would be equivalent to $\tanh (W)$ for a single matrix $W$. When our modification is applied to an LSTM cell, we obtain a Multi-Weight LSTM cell with the update equations

$$
\begin{aligned}
i_{t} & =\sigma\left(W_{\mathrm{ix}} x_{t}+W_{\mathrm{ih}} h_{t-1}+b_{\mathrm{i}}\right), \\
f_{t} & =\sigma\left(W_{\mathrm{fx}} x_{t}+W_{\mathrm{fh}} h_{t-1}+b_{\mathrm{f}}\right), \\
o_{t} & =\sigma\left(W_{\mathrm{ox}} x_{t}+W_{\mathrm{oh}} h_{t-1}+b_{\mathrm{o}}\right), \\
c_{t} & =f_{t} \odot c_{t-1}+\sum_{i=1}^{K} p_{i} \odot \tanh \left(W_{\mathrm{cx}}^{i} x_{t}+W_{\mathrm{ch}}^{i} h_{t-1}+b^{i}\right), \\
h_{t} & =o_{t} \odot c_{t},
\end{aligned}
$$

where $K$ is the number of weight matrices. Our strategy is to extend the LSTM cell with a gate control mechanism to allow

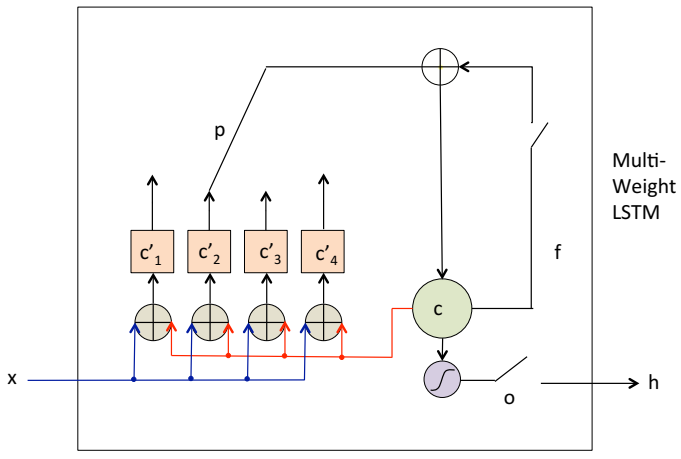

Figure 2: The structure of a multi-weight LSTM cell.

it to choose between two or more matrices when updating the state vector for multi-faceted data (see Fig. 2).

As another example, when applied to the GRU cell architecture, we obtain cell architecture of the form

$$
\begin{aligned}
r_{t} & =\sigma\left(W_{\mathrm{rx}} x_{t}+W_{\mathrm{rh}} h_{t-1}+b_{\mathrm{r}}\right), \\
u_{t} & =\sigma\left(W_{\mathrm{ux}} x_{t}+W_{\mathrm{uh}} h_{t-1}+b_{\mathrm{u}}\right), \\
v_{t}^{i} & =\tanh \left[W_{\mathrm{vx}}^{i} x_{t}+W_{\mathrm{vr}}^{i}\left(r_{t} \odot h_{t-1}\right)+b_{\mathrm{v}}^{i}\right], 1 \leq i \leq K \\
o_{t} & =h_{t}=u_{t} \odot h_{t-1}+\left(1-u_{t}\right) \odot \sum_{i=1}^{K} p_{i} v_{t}^{i},
\end{aligned}
$$

where the $v_{i}^{t}$ are the activation vectors for the GRU. The subscript of $W$ indicates the weight of the connection between two gates, and the superscript of $W$ indicates which matrix is being used. For simplicity, in later experiments, we will consider the modified LSTM cell architecture and take $K=2$. Other cell structures improve to a similar degree.

While our architecture bears some superficial resemblance to ensemble methods, there are a few crucial differences. First, while ensemble methods aggregate the final outputs that correspond to different internal states [Zhang et al., 2016], our model uses a single RNN with only one internal state. Internally, the decisions for arriving at this internal state are based on multiple matrices. This novel approach is geared at multi-faceted data. Second, while ensemble methods take an average of several model outputs, our model relies on a weighted average over the matrices, for which the weights are learnt during training.

Finally, we explain how the $p_{i}$ are computed. The weights of the matrices, $p_{i}$, are also learnt using the input word and the state vector,

$$
p_{i}^{\prime}=h_{p}^{i}(x, c)
$$

and then go through a softmax normalization,

$$
p_{i}=\frac{\exp \left(p_{i}^{\prime}\right)}{\sum_{j} \exp \left(p_{j}^{\prime}\right)} .
$$

A detailed description of our scheme is shown in Fig. 2. In our implementation, we choose $h_{p}^{i}$ such that

$$
p_{i}^{\prime}=W_{\mathrm{px}}^{i} x+W_{\mathrm{pc}}^{i} c+b_{\mathrm{p}}^{i} .
$$




\subsection{Objective function}

Since the objective function is dependent on the task, we discuss a series of three example tasks (which are explained in more detail in Section 4).

1. Language modeling: Here, the loss function is set to

$$
L=-\sum_{t=1}^{T} \log p\left(w_{t} \mid w_{1}, \ldots, w_{t-1}\right) .
$$

This loss function can be understood as maximizing the probability of a sentence.

2. Cloze tests: In this task, we set the output layer to be a conditional random field (CRF). The objective function is the conditional probability of the true answer to the cloze test, i.e.,

$$
L=-\log p\left(w_{t} \mid w_{1}, \ldots, w_{t-1}, w_{t+1}, \ldots, w_{N}\right),
$$

where $w_{t}$ is the blank word to be filled.

3. Character to word embedding: Here, we choose the loss function to be the cosine similarity of the ground truth word embedding and the one generated by our model from character embeddings. More precisely, we have

$$
L=-\left\langle v_{w}, v_{c}\right\rangle,
$$

where the minus sign is placed to maximize the cosine similarity, $v_{w}$ is the genuine word embedding (which serves as the ground truth target), and $v_{c}$ is the word embedding generated from character embeddings.

\subsection{Training}

We use mini-batch stochastic gradient descent to train our model. The learning rate is controlled by AdaDelta [Zeiler, 2012]. As before, we distinguish three cases:

1) Language modeling: The parameters are learnt through the following equation

$$
\frac{\partial L}{\partial \theta}=\sum_{k} \frac{\partial L}{\partial w_{k}} \frac{\partial w_{k}}{\partial \theta}
$$

where $\theta$ refers to the parameters.

2) Cloze tests: The training for cloze test tasks is similar to language modeling, except that here, only one word is being predicted. Hence the parameters are updated according to

$$
\frac{\partial L}{\partial \theta}=\frac{\partial L}{\partial w_{t}} \frac{\partial w_{t}}{\partial \theta}
$$

where $\theta$ is the parameter and $w_{t}$ is the word being predicted.

3) Character to word embedding: Denote the character embeddings as $c_{1}, c_{2}, \ldots, c_{n}$. According to the chain rule, the parameters are updated by

$$
\frac{\partial L}{\partial \theta}=\frac{\partial L}{\partial v_{c}} \sum_{k} \frac{\partial v_{c}}{\partial c_{k}} \frac{\partial c_{k}}{\partial \theta},
$$

where $\theta$ is, as usual, a parameter.

For all three cases, the gradient updates depend on the $p_{i}$ values, and we use standard backpropagation along the computation graph.

\begin{tabular}{ccc}
\hline \hline & Perplexity & ROUGE2 \\
\hline GRU & 123.0 & 12.1 \\
MuFuRu & 119.7 & 14.7 \\
Our model & 101.4 & 17.2 \\
\hline
\end{tabular}

Table 1: Experiment Results of Language Modeling.

\section{Empirical Evaluation}

We evaluate our model on a range of different sequential learning tasks, in order to demonstrate the improvements obtained with our enhanced model.

\subsection{Language Modeling}

We use the standard Penn Treebank dataset [Marcus et al., 1993] for language modeling. To measure the effectiveness of our language models, we consider the standard perplexity and ROUGE2 measures. Recall that perplexity is defined as

$$
\text { Perplexity }=\exp \left(\frac{1}{N} \sum_{i=1}^{N}-\log p\left(w_{i} \mid \cdots, w_{i-1}\right)\right),
$$

where $w_{i}$ is the $i$-th word and $N$ is the length of the sentence. ROUGE2 is defined as the recall over bigrams. We compare our model with two strong baselines, GRUs and $\mathrm{Mu}-$ FuRu [Weissenborn and Rocktäschel, 2016]. At test time, we use beam search to find the best word sequence. The results are given in Table 1. Compared against the baselines, our model achieves the best performance both in terms of perplexity and in terms of the ROUGE2 measure. Our model reduces the perplexity of GRUs by $21.3 \%$ and that of MuFuRUs by $18.0 \%$. At the same time, our model improves by $42 \%$ over GRUs and by $17 \%$ over MuFuRUs in terms of the respective ROUGE2 scores.

Additionally, we evaluate our model on two further datasets: (1) Restaurant Reservation Dialogs [Eric and Manning, 2017] and (2) the Switchboard Dialog Act Corpus [Liu and Lane, 2017]. The first dataset consists of raw dialogue text and system commands. The dialogue contains both user and system utterances. The system commands are used to query its knowledge base in order to respond to a users' utterance. The average number of utterances in each dialogue is 14 . The vocabulary size is 1,229 . The dataset is split into training set, test set, and validation set, with 1,618, 1,117, and 500 dialogues, respectively. In the second (Switchboard) dataset, there are part-of-speech (POS) tags associated with the utterances. The data is split into 14 equal parts, 10 of which are used for training, one for testing, and the remaining three for validation. In total, there are $223.5 \mathrm{~K}$ utterances in the corpus. Only the most frequent $10 \mathrm{~K}$ words are considered for the vocabulary.

For the restaurant reservation dialog dataset, the previous state-of-the-art [Eric and Manning, 2017] achieves an accuracy of $48 \%$, while our result is $51.3 \%$. Our model improves the accuracy of the previous state-of-the-art by $6.8 \%$. The performance comparison for the Switchboard Dialog Act Corpus is shown in Table 2. Our model reduces the perplexity of the previous state-of-the-art [Liu and Lane, 2017] by 8.8\%. 
Proceedings of the Twenty-Sixth International Joint Conference on Artificial Intelligence (IJCAI-17)

\begin{tabular}{cc}
\hline \hline & Perplexity \\
\hline Previous state-of-the-art & 58.4 \\
Our result & 53.7 \\
\hline
\end{tabular}

Table 2: Experiment Results of Dialogue Corpora.

\begin{tabular}{cc}
\hline \hline Algorithms & Accuracy \\
\hline Attentive reader & 0.53 \\
Stanford reader & 0.64 \\
AS reader & 0.57 \\
GA reader & 0.57 \\
Our model & 0.69 \\
\hline
\end{tabular}

Table 3: Experiment Results of Cloze tests.

\subsection{Cloze Test}

In a Cloze test, one is given a sentence, from which one of the words has been excised and left blank. The goal is to fill the blank with an appropriate word, choosing among different options. We use the "Who-did-What" dataset [Onishi et al., 2016], which consists of around 0.2 million cloze questions. On average, there are 3.5 different choices for each blank. The total vocabulary size is more than 0.3 million. We compare with four neural network baselines:

1. Attentive Reader [Hermann et al., 2015]: LSTM with attention mechanism.

2. Stanford Reader [Chen et al., 2016]: As above, but with a bilinear term.

3. Attention Sum (AS) Reader [Kadlec et al., 2016]: GRU with attention.

4. Gated-Attention (GA) Reader [Dhingra et al., 2016]: As above, but with gated layers.

The accuracy scores of these baselines and our model are shown in Table 3. It can be seen that our model is $5 \%$ better than the best baseline on the "Who-did-What" dataset.

\subsection{Character-Level Word Embedding}

In this subsection, we evaluate on the character-to-word embedding task, a supervised task, where the goal is to predict a word embedding given a sequence of character embeddings. There are several challenges. First, each word has a different number of characters. This is naturally addressed by using the RNN structure, which is well-suited for variable-length inputs. Second, the dimensions of the character embedding and the word embedding diverge significantly (the former is around 10, while the latter is around 400). For the second problem, we use a non-square matrix to transform the input vector to the state vector, and another non-square matrix to transform the state vector to the output vector.

The intuition for why our model may work better for this character-to-word task than previous methods is that the data may be bimodal in the sense that there are two kinds of words. As a concrete example, consider the words "inconsiderable" and "piano". While the semantics of the former is reasonably predictable from the standard morphological rules of the English language, the latter is borrowed from a foreign language. This shows that there are at least two logics inherent to the data, which can easily be captured by our multi-weight model, but is challenging for previous methods.

We compare our model with the model of Santos et al. [2014] and use the Penn Treebank dataset (standard Wall Street Journal portion) [Marcus et al., 1993]. The ground truth word embedding is generated using the standard word2vec tool using SGNS [Mikolov et al., 2013]. The accuracies of Santos et al. and of our model are $97.3 \%$ and $98.1 \%$, respectively. Again, we find that our model outperforms previous work; in this case, the method of Santos et al. by $0.8 \%$ (absolute).

\subsection{Analysis and Discussion}

We next shed further light on why and how our multi-weight approach succeeds, while also providing an error analysis.

Theoretical Analysis. For simplicity, we assume there is a mixing of two separated tasks and set the number of matrices to two. The case of more than two matrices is analogous. In particular, we consider the instructive example of interleaving English speech generation and French speech generation, as is common in code-switching and in multilingual announcements.

Initially, the parameters in our model will be quite random. During training, the weight matrices and state vectors will stabilize to a steady state. In this steady state, ideally, each character within an English utterance would result in selecting the weight matrix $W_{1}$, while each character within a French utterance would select for the weight matrix $W_{2}$. In this case, our model can be understood as a seamless combination of two language models, the English model and the French model. This explains our model's ability to deal with data in which different aspects are blended together, and its ability to switch between them.

The reader may have noticed that similar mechanisms also appear in a different form in attention models. However, there are a few notable differences. First, in an attention model, a weight is used to determine to what extent to consider the hidden state obtained for a given input, e.g. different keywords in a sentence [Wang et al., 2016], while in our multiweight LSTM model, the focus is instead on choosing the right weight matrices to produce a hidden state. Second, for attention models, the steps that require attention are usually fixed, while in our model, the number of selections may vary for different instances. Third, attention mechanisms typically are used to enable each output step to focus on the hidden states for different parts of an input sequence. Our gating mechanism instead enables entirely different operations, not just varying attention, and enables this for every input step, while an attention model applies only after the entire input has been consumed. Fourth, unlike attention mechanisms, the weight matrices for these different operations are automatically learned from the data.

Experimental Analysis. In order to validate these theoretical intuitions experimentally, we create two artificial datasets. The first of these consists of the union of a large biomedical corpus [Bunescu and Mooney, 2005] and a news corpus [Das 


\begin{tabular}{cc}
\hline \hline Bio & News \\
0.81 & 0.27 \\
\hline English & French \\
0.15 & 0.93 \\
\hline
\end{tabular}

Table 4: The relation between the selected weight matrix and the type of sentence.

\begin{tabular}{cc}
\hline \hline Error class & Percentage \\
\hline Infrequent Mechanism & $63 \%$ \\
Prior Knowledge & $21 \%$ \\
Others & $16 \%$ \\
\hline
\end{tabular}

Table 5: Distribution of different error classes.

and Bandyopadhyay, 2010], with a randomly shuffled sentence order. In the second dataset, we take an English corpus [Marcus et al., 1993] and another French corpus [Degand and Bestgen, 2003], and again randomly shuffle the sentences.

Now, we examine and interpret the cell behavior on these manufactured datasets. In our experiment, we again set the number of weight matrices to be two, since it suffices for capturing the bimodal nature of these datasets. After training our model and before prediction, we keep track of which corpus each test sentence originated from and trace the value of $p_{i}$ in Eq. (8) of the cell. Recall that $p_{i}$ denotes the probability of selecting the $i$-th weight matrix $W_{i}$.

In Table 4, we list the corresponding $p_{1}$ for the respective two corpora in the two datasets. Since, in this two-weight matrix instantiation of multi-weight LSTMs, $p_{2}=1-p_{1}$ can be determined from $p_{1}$, we omit its values in the table. It can be seen that, in both datasets, one kind of data selects $W_{1}$ much more often, while the other goes through $W_{2}$ much more often.

Error Analysis. To further improve the architecture of our framework in the future, it is helpful to scrutinize the failure cases of our models. In particular, we have categorized the failure cases observed for the cloze tests into the following categories.

1. Infrequent Mechanism. For example, the choices for the blank in "... Sources close to the presidential palace said that Fujimori declined at the last moment to leave the country and instead he will send a high level delegation to the ceremony, at which Chilean President Eduardo Frei will pass the mandate to __ . ..." are (1) Augusto Pinochet, (2) Jack Straw, and (3) Ricardo Lagos. Our model wrongly chooses option (2), while the correct answer is choice (1). The model may have misunderstood that Eduardo Frei is in close contact with Jack Straw, since the name Jack Straw is closest to Eduardo Frei among the three choices. Such errors likely occur because this is an infrequent case not covered by the main mechanisms of inference learnt by our model.

2. Prior Knowledge: For example, the choices for the blank in "... U.S. officials, repeatedly stymied in the quest to oust alleged war criminals from the Bosnian Serb leadership, said Tuesday that increased NATO patrols could improve the chances that indicted suspects such as Radovan Karadzic and ___ will be arrested. ..." are (1) Warren Christopher, (2) Nicholas Burns, (3) George Joulwan, (4) Simon Haselock, and (5) Ratko Mladic. Our model wrongly chooses option (3), while the correct answer is choice (5). It is not mentioned that Ratko Mladic is a criminal in the context, though it is common knowledge, and hence the model may not have captured that the word "suspect" hints at the blank being filled with this name. Overcoming such errors may require an additional dictionary or encyclopedia.

The relative percentages of different classes of errors are given in Table 5. For this evaluation, we randomly select 100 failure cases and categorize them manually. It can be seen that the class of Infrequent Mechanism errors occupies the largest portion, followed by Prior Knowledge, which comes second. Thus, focusing on avoiding these two types of errors may greatly enhance the success rate of our model on cloze test scores specifically and other tasks in general.

\section{Conclusion}

In summary, we have proposed a novel framework for multiweight RNN cells that rely on multiple weight matrices to better cope with multi-faceted data. The mechanism is explained theoretically and experimentally analyzed in substantial detail. Extensive experiments on multiple tasks show the consistent superiority of our model against previous state-ofthe-art models. Since most real-world data is multi-faceted in nature, we expect this approach to be of broad applicability in numerous different tasks.

\section{References}

[Bengio and others, 2009] Yoshua Bengio et al. Learning deep architectures for ai. Foundations and trends $\mathrm{Q}$ in $\mathrm{Ma}$ chine Learning, 2(1):1-127, 2009.

[Bengio et al., 1994] Yoshua Bengio, Patrice Simard, and Paolo Frasconi. Learning long-term dependencies with gradient descent is difficult. IEEE transactions on neural networks, 5(2):157-166, 1994.

[Bunescu and Mooney, 2005] Razvan C. Bunescu and Raymond J. Mooney. Subsequence kernels for relation extraction. Advances in Neural Information Processing Systems, pages 171-178, 2005.

[Caruana, 1997] Rich Caruana. Multitask learning. Machine Learning, 28(1):41-75, 1997.

[Chen et al., 2016] Danqi Chen, Jason Bolton, and Christopher D Manning. A thorough examination of the $\mathrm{cnn}$ /daily mail reading comprehension task. arXiv preprint arXiv:1606.02858, 2016.

[Cho et al., 2014] Kyunghyun Cho, Bart Van Merriënboer, Dzmitry Bahdanau, and Yoshua Bengio. On the properties of neural machine translation: Encoder-decoder approaches. arXiv preprint arXiv:1409.1259, 2014. 
[Chung et al., 2014] Junyoung Chung, Caglar Gulcehre, KyungHyun Cho, and Yoshua Bengio. Empirical evaluation of gated recurrent neural networks on sequence modeling. arXiv preprint arXiv:1412.3555, 2014.

[Das and Bandyopadhyay, 2010] D. Das and S. Bandyopadhyay. Sentence level emotion tagging on blog and news corpora : Journal of intelligent systems. Journal of Intelligent Systems, 19(2):145-162, 2010.

[Degand and Bestgen, 2003] Liesbeth Degand and Yves Bestgen. Towards automatic retrieval of idioms in french newspaper corpora. Literary \& Linguistic Computing, 18:249-259, 2003.

[Dhingra et al., 2016] Bhuwan Dhingra, Hanxiao Liu, William W Cohen, and Ruslan Salakhutdinov. Gatedattention readers for text comprehension. arXiv preprint arXiv:1606.01549, 2016.

[Eric and Manning, 2017] Mihail Eric and Christopher D Manning. A copy-augmented sequence-to-sequence architecture gives good performance on task-oriented dialogue. arXiv preprint arXiv:1701.04024, 2017.

[Fausett, 1994] Laurene V Fausett. Fundamentals of neural networks. Prentice-Hall, 1994.

[Glorot et al., 2011] Xavier Glorot, Antoine Bordes, and Yoshua Bengio. Domain adaptation for large-scale sentiment classification: A deep learning approach. In Proceedings of the 28th international conference on machine learning (ICML-11), pages 513-520, 2011.

[Greff et al., 2016] Klaus Greff, Rupesh K Srivastava, Jan Koutník, Bas R Steunebrink, and Jürgen Schmidhuber. Lstm: A search space odyssey. IEEE transactions on neural networks and learning systems, 2016.

[Hermann et al., 2015] Karl Moritz Hermann, Tomas Kocisky, Edward Grefenstette, Lasse Espeholt, Will Kay, Mustafa Suleyman, and Phil Blunsom. Teaching machines to read and comprehend. In Advances in Neural Information Processing Systems, pages 1693-1701, 2015.

[Hochreiter and Schmidhuber, 1997] Sepp Hochreiter and Jürgen Schmidhuber. Long short-term memory. Neural computation, 9(8):1735-1780, 1997.

[Jozefowicz et al., 2015] Rafal Jozefowicz, Wojciech Zaremba, and Ilya Sutskever. An empirical exploration of recurrent network architectures. In Proceedings of the $32 N d$ International Conference on International Conference on Machine Learning - Volume 37, ICML'15, pages 2342-2350. JMLR.org, 2015.

[Kadlec et al., 2016] Rudolf Kadlec, Martin Schmid, Ondrej Bajgar, and Jan Kleindienst. Text understanding with the attention sum reader network. arXiv preprint arXiv:1603.01547, 2016.

[Le et al., 2015] Quoc V Le, Navdeep Jaitly, and Geoffrey E Hinton. A simple way to initialize recurrent networks of rectified linear units. arXiv preprint arXiv:1504.00941, 2015.
[Liu and Lane, 2017] Bing Liu and Ian Lane. Dialog context language modeling with recurrent neural networks. arXiv preprint arXiv:1701.04056, 2017.

[Marcus et al., 1993] Mitchell P Marcus, Mary Ann Marcinkiewicz, and Beatrice Santorini. Building a large annotated corpus of english: the penn treebank. Computational Linguistics, 19(2):313-330, 1993.

[Mikolov and Zweig, 2012] Tomas Mikolov and Geoffrey Zweig. Context dependent recurrent neural network language model. SLT, 12:234-239, 2012.

[Mikolov et al., 2010] Tomas Mikolov, Martin Karafit, Lukas Burget, Jan Cernock, and Sanjeev Khudanpur. Recurrent neural network based language model. In INTERSPEECH 2010, Conference of the International Speech Communication Association, Makuhari, Chiba, Japan, September, pages 1045-1048, 2010.

[Mikolov et al., 2013] Tomas Mikolov, Kai Chen, Greg Corrado, and Jeffrey Dean. Efficient estimation of word representations in vector space. arXiv preprint arXiv:1301.3781, 2013.

[Mikolov et al., 2014] Tomas Mikolov, Armand Joulin, Sumit Chopra, Michael Mathieu, and Marc'Aurelio Ranzato. Learning longer memory in recurrent neural networks. arXiv preprint arXiv: 1412.7753, 2014.

[Onishi et al., 2016] Takeshi Onishi, Hai Wang, Mohit Bansal, Kevin Gimpel, and David McAllester. Who did what: A large-scale person-centered cloze dataset. arXiv preprint arXiv:1608.05457, 2016.

[Santos and Zadrozny, 2014] Cicero Nogueira Dos Santos and Bianca Zadrozny. Learning character-level representations for part-of-speech tagging. In International Conference on Machine Learning, pages 1818-1826, 2014.

[Wang and Cho, 2015] Tian Wang and Kyunghyun Cho. Larger-context language modelling. arXiv preprint arXiv:1511.03729, 2015.

[Wang et al., 2016] Linlin Wang, Zhu Cao, Gerard de Melo, and Zhiyuan Liu. Relation classification via multi-level attention CNNs. In Proceedings of ACL 2016, 2016.

[Weissenborn and Rocktäschel, 2016] Dirk Weissenborn and Tim Rocktäschel. Mufuru: The multi-function recurrent unit. arXiv preprint arXiv:1606.03002, 2016.

[Zeiler, 2012] Matthew D. Zeiler. Adadelta: An adaptive learning rate method. Computer Science, 2012.

[Zhang et al., 2016] Dong Zhang, Shoushan Li, Hongling Wang, and Guodong Zhou. User classification with multiple textual perspectives. In Proceedings of COLING, pages 2112-2121, 2016.

[Zhou et al., 2016] Guo-Bing Zhou, Jianxin Wu, Chen-Lin Zhang, and Zhi-Hua Zhou. Minimal gated unit for recurrent neural networks. International Journal of Automation and Computing, 13(3):226-234, 2016. 